\title{
The Second Bounded Cohomology of 3-manifolds
}

\author{
By
}

Koji FuJIwarA* and Ken'ichi OHshikA**

\begin{abstract}
Let $G$ be the fundamental group of a compact, orientable 3-manifold $M$. We show that if each piece of the canonical decomposition of $M$ has a geometric structure (e.g. when $G$ contains $\mathbb{Z}^{2}$ ), then either $G$ has infinite dimensional second bounded cohomology or $G$ is virtually solvable.
\end{abstract}

\section{$\S 1$. Introduction}

The bounded cohomology of a discrete group $G$ is defined using a subcomplex of the ordinary cochain complex ([G1]). Set

$$
C_{b}^{k}(G ; \mathbb{R})=\left\{f: G^{k} \rightarrow \mathbb{R} \mid f \text { has bounded range }\right\}
$$

A coboundary operator $\delta: C_{b}^{k}(G ; \mathbb{R}) \rightarrow C_{b}^{k+1}(G ; \mathbb{R})$ is given by

$$
\begin{aligned}
\delta f\left(g_{0}, \ldots, g_{k}\right)= & f\left(g_{1}, \ldots, g_{k}\right)+\sum_{i=1}^{k}(-1)^{i} f\left(g_{0}, \ldots, g_{i-1} g_{i}, \ldots, g_{k}\right) \\
& +(-1)^{k+1} f\left(g_{0}, \ldots, g_{k-1}\right) .
\end{aligned}
$$

Communicated by K. Saito. Received September 6, 2001.

2000 Mathematics Subject Classification(s): Primary 57N10; Secondary 20F65, 57M07. Key words and phrases: Bounded cohomology, 3-manifold groups, Haken manifolds.

This collaboration has started while the authors were visiting MSRI. The first author was supported in part by NSF grant DMS-9022140 and a JSPS grant.

* Mathematical Institute, Tohoku University, Sendai 980-8578, Japan.

e-mail: fujiwara@math.tohoku.ac.jp

** Department of Mathematics, Graduate School of Science, Osaka University, Toyonaka, Osaka 560-0043, Japan.

e-mail: ohshika@math.wani.osaka-u.ac.jp 
The cohomology of the complex $\left\{C_{b}^{k}(G ; \mathbb{R}), \delta\right\}$ is called the (real) bounded cohomology group of $G$, and denoted by $H_{b}^{*}(G ; \mathbb{R})$.

Gromov gave a definition of the bounded cohomology for topological spaces, and showed that it is isomorphic to the one for its fundamental group for a large class of spaces including all manifolds (not necessary $K(\pi, 1)$ ). It is known that $H_{b}^{1}(G ; \mathbb{R})$ is trivial for every group $G$, and that $H_{b}^{n}(G ; \mathbb{R})$ is trivial for all $n \geq 1$ if $G$ is amenable. There is a nice account on the subject by Ivanov $[\mathrm{I}]$.

Brooks $[\mathrm{B}]$ constructed countably many independent second bounded cohomology classes for free groups $G$ of rank at least two (see also [Mi]). He used the canonical action of a free group on a simplicial tree. His idea applies to a group which acts on a geodesic space that is "hyperbolic" in the sense of Gromov [G2] ([BaGh], [EF], [F1], [F2]). The group $G$ in the following list has infinite dimensional $H_{b}^{2}(G ; \mathbb{R})$. We remark that if the dimension of $H_{b}^{2}(G ; \mathbb{R})$ is infinite, then it is automatically the cardinal of the continuum since $H_{b}^{2}(G ; \mathbb{R})$ is a Banach space ([I], [MaMo]).

\section{List.}

(a) Non-elementary word-hyperbolic groups ([EF]). (See [G2] for the definition of word-hyperbolic groups): e.g. free groups of rank at least two, the surface groups of genus at least two, and the fundamental group of a closed Riemannian manifold of negative sectional curvature.

(b) Groups $G$ which decompose as $G=A *_{C} B$ such that $|C \backslash A / C| \geq 3$ and $|B / C| \geq 2$ or $G=A *_{C, \varphi}$ such that $|A / C| \geq 2$ and $|A / \varphi(C)| \geq 2([\mathrm{~F} 1])$.

(c) Groups $G$ which act properly discontinuously by isometries on a Gromovhyperbolic space such that the limit set of the action has more than two points ([F2]): e.g. lattices in a rank-1 semi-simple Lie group, Kleinian groups which are not virtually nilpotent.

We apply those results to the fundamental group of a compact orientable 3-manifold. A group $G$ is called virtually solvable if it contains a solvable subgroup of finite index. In particular $H_{b}^{2}(G ; \mathbb{R})$ is then trivial. The following is our main theorem.

Theorem 1.1 (Algebraic). Let $G$ be the fundamental group of a compact, orientable 3-manifold $M$. Suppose that $G$ decomposes non-trivially as $G=A *_{C} B$ or $A *_{C}$. Then either $H_{b}^{2}(G ; \mathbb{R})$ is infinite dimensional or $G$ is virtually solvable. 
Moreover $G$ is virtually solvable if and only if $G$ is isomorphic to either $\mathbb{Z}$ or $\mathbb{Z}_{2} * \mathbb{Z}_{2}$ or the fundamental group of one of the following manifolds.

(i) A torus bundle over $S^{1}$.

(ii) A Seifert fibred space with a Euclidean base orbifold.

(iii) A manifold obtained by gluing two twisted $S^{1}$-bundles over Möbius bands along their boundaries. Then $G$ admits an exact sequence

$$
1 \rightarrow \mathbb{Z} \times \mathbb{Z} \rightarrow G \rightarrow \mathbb{Z}_{2} * \mathbb{Z}_{2} \rightarrow 1
$$

Theorem 1.1 applies to all Haken manifolds. More generally one can show the following.

Theorem 1.2 (Topological). Let $G$ be the fundamental group of a compact orientable 3-manifold $M$. Suppose that $M$ has no boundary component homeomorphic to $S^{2}$. Assume that each piece of the canonical decomposition of $M$ has a geometric structure. Then $H_{b}^{2}(G ; \mathbb{R})$ is infinite dimensional or $G$ is virtually solvable.

Moreover, $G$ is virtually solvable if and only if $M$ is homeomorphic to one of the manifolds in (i), (iii) in Theorem 1.1, or one of the following.

(ii)' A Seifert fibred space with a Euclidean or spherical base orbifold.

(iv) A manifold which is finitely covered by $S^{3}$. (Then $G$ is finite).

(v) $S^{2} \times S^{1}$ and $D^{2} \times S^{1}$. (Then $\left.G \cong \mathbb{Z}\right)$.

(vi) $P R^{3} \sharp P R^{3}$. (Then $\left.G \cong \mathbb{Z}_{2} * \mathbb{Z}_{2}\right)$.

The geometrization conjecture ([T]) says that if $M$ is a compact orientable 3-manifold, each piece of the canonical decomposition of $M$ has a geometric structure (see Section 2).

Corollary 1.3. Let $G$ be the fundamental group of a compact, orientable 3-manifold $M$. If $G$ contains $\mathbb{Z} \times \mathbb{Z}$, then $H_{b}^{2}(G ; \mathbb{R})$ is infinite dimensional or $G$ is virtually solvable.

It follows that if $G$ is a knot group, then $H_{b}^{2}(G ; \mathbb{R})$ is infinite dimensional or the knot is trivial ([F2]).

Theorem 1.2 or Corollary 1.3 applies to any compact orientable 3 -manifold $M$ unless it is irreducible, non-Haken (in particular closed) with infinite fundamental group $G$ without $\mathbb{Z} \times \mathbb{Z}$ subgroups. Hyperbolization conjecture ([T]) says that such 3 -manifold is hyperbolic, so that $G$ is word-hyperbolic. 
Let $M$ be an orientable, non-compact 3-manifold. If the fundamental group is finitely generated, then $M$ has a compact submanifold, called a core, which is homotopy equivalent to $M([\mathrm{Sc} 2])$. One can apply our results to a core of $M$.

\section{$\S 2$. Canonical Decompositions of 3-manifolds}

We review standard facts about 3-manifolds (see [Sc1]). Let $M$ be a compact orientable 3 -manifold with no boundary component homeomorphic to a 2-sphere. A 2-sphere embedded in $M$ is called essential unless it bounds a 3-ball in $M$. Consider a maximal system (always finite) of disjoint, non-parallel, essential, separating, embedded spheres $\left\{S_{i}\right\}$ in $M$. Cutting $M$ along $\left\{S_{i}\right\}$ gives a connected sum decomposition such that each summand does not contain essential, separating, embedded spheres (called prime). The decomposition is unique and called the prime decomposition of $M$.

Let $M$ be a compact, orientable, prime 3-manifold. Unless $M$ is $S^{2} \times S^{1}$, $M$ does not contain a non-separating embedded 2-sphere. Such manifolds are called irreducible.

Let $M$ be a compact, orientable, irreducible 3-manifold. If $\partial M$ is compressible in $M$, then by the loop theorem, one can find a compressing disc $D$ for $\partial M$. One cuts $M$ along $D$ and obtains $N$. If $\partial N$ is still compressible, we continue the same operation. This process must stop in finite steps and gives a splitting of $M$ into pieces with incompressible boundary.

Let $M$ be a compact, orientable, irreducible, 3-manifold with possibly empty incompressible boundary. An embedded torus in a 3-manifold is said to be essential if it is incompressible and not parallel to a boundary component. The theory of Jaco-Shalen-Johannson ([JSh], [Jo]) says that there is a finite collection of disjoint, incompressible, embedded tori $\left\{T_{i}\right\}$ such that by cutting $M$ along $T_{i}$, we obtain a family of 3-manifolds each of which is either a Seifert fibred space or admits no essential tori (called atoroidal). A Seifert fibred space is an $S^{1}$-bundle whose base space is a 2 -orbifold. The process and its outcome we described of cutting $M$ along embedded 2-spheres, 2-discs, and tori is called a canonical decomposition.

A locally homogeneous Riemannian metric on a manifold with a certain maximal property is called a geometric structure. In dimension 3 , there are eight geometric structures which are modeled on $\mathbf{S}^{3}, \mathbf{E}^{3}, \mathbf{H}^{3}, \mathbf{H}^{2} \times \mathbf{E}, \mathbf{S}^{2} \times$ E, $\widetilde{S L_{2}(\mathbb{R})}, N i l$, and $S o l v$, respectively.

The geometrization conjecture $([\mathrm{T}])$ is that each piece in the canonical decomposition admits a geometric structure. It is known that the Seifert fibred 
spaces admit geometric structures. A compact irreducible 3-manifold is called Haken if it contains a properly embedded two-sided incompressible surface. By Thurston's uniformization theorem, every atoroidal, Haken manifold admits a geometrically finite hyperbolic structure on its interior (called hyperbolic).

\section{$\S 3 . \quad$ Proofs}

The following result by Bouarich is useful.

Theorem $3.1([\mathrm{Bo}])$. Let $G_{3} \rightarrow G_{2} \rightarrow G_{1} \rightarrow 1$ be an exact sequence of groups. Then the induced sequence of the second bounded cohomology is exact;

$$
0 \rightarrow H_{b}^{2}\left(G_{1} ; \mathbb{R}\right) \rightarrow H_{b}^{2}\left(G_{2} ; \mathbb{R}\right) \rightarrow H_{b}^{2}\left(G_{3} ; \mathbb{R}\right) .
$$

In particular $H_{b}^{2}\left(G_{1} ; \mathbb{R}\right)$ and $H_{b}^{2}\left(G_{2} ; \mathbb{R}\right)$ are isomorphic if $G_{3}$ is amenable.

We prove Theorems 1.1 and 1.2 simultaneously.

Proof of Theorems 1.1 and 1.2. We start with the assumption of Theorem 1.1. We may assume that there is no connected component of $\partial M$ homeomorphic to $S^{2}$. We do not lose generality since we can change $M$ by filling in 3-balls without changing $G$.

Let $M=M_{1} \sharp \cdots \sharp M_{n}$ be the prime decomposition of $M$. For our purpose, we may assume that $\pi_{1}\left(M_{i}\right)$ is not trivial for each $i$. If the prime decomposition of $M$ has more than one summands, then $G$ is freely decomposable. It follows from List (b) that $H_{b}^{2}(G ; \mathbb{R})$ is infinite dimensional unless $G$ is isomorphic to $\mathbb{Z}_{2} * \mathbb{Z}_{2}$.

Let $M$ be prime. If $M$ is $S^{2} \times S^{1}$, then $G \cong \mathbb{Z}$ and we are done. Therefore we suppose that $M$ is irreducible. If there is a component of $\partial M$ which is not $\pi_{1}$-injective, then by the loop theorem, $G$ decomposes as $G=A *_{\{e\}}$ or $G=A * B$. In the first case $H_{b}^{2}(G)$ is infinite dimensional unless $G \cong \mathbb{Z}$ (List (b)). We have already discussed the second case. Therefore we suppose that $M$ is boundary irreducible. ( $\partial M$ might be empty). Then $M$ is Haken. Indeed, if $\partial M$ is not empty, then $M$ is Haken. If $M$ is closed, by a standard technique in 3 -dimensional topology, it follows from the assumption that $G=A *_{C} B$ or $G=$ $A *_{C}$ and that one can find a closed, embedded, 2-sided, incompressible surface $F$ in $M$ so that $\pi_{1}(F) \subset C$. Since $M$ is irreducible, $F$ is not homeomorphic to $S^{2}$, hence $M$ is Haken.

If $M$ is Haken, each piece of the canonical decomposition of $M$ admits a geometric structure, which is the assumption in Theorem 1.2. Now we start the proof of Theorem 1.2. At the end we obtain Theorem 1.1 too. We see 
that $G$ is either finite or isomorphic to $\mathbb{Z}$, or isomorphic to $\mathbb{Z}_{2} * \mathbb{Z}_{2}$ if and only if $M$ is homeomorphic to the manifolds in (iv), (v), or (vi) in Theorem 1.2, respectively.

In the rest, we assume that $M$ is irreducible with (possibly empty) incompressible boundary. If the torus decomposition of $M$ is trivial, $M$ is homeomorphic to one of the following manifolds.

(1) A torus bundle over $S^{1}$.

(2) A Seifert fibred space with a spherical or Euclidean base orbifold. (If $M$ is Haken, a spherical base does not appear.)

(3) A Seifert fibred space with a hyperbolic base orbifold $O$.

(4) A geometrically finite hyperbolic manifold (we consider the interior of $M$ ).

In the case of (1) or (2), $G$ is virtually solvable. In the case of (3), there is an exact sequence

$$
1 \rightarrow \mathbb{Z} \rightarrow G \rightarrow \pi_{1}^{\text {orb }}(O) \rightarrow 1
$$

Since $H_{b}^{2}(G) \cong H_{b}^{2}\left(\pi_{1}^{\text {orb }}(O)\right)$, (by Theorem 3.1) and $\pi_{1}^{\text {orb }}(O)$ is a nonelementary word-hyperbolic group, $H_{b}^{2}(G)$ is infinite dimensional (List (a)). For (4), since $G$ is a Kleinian group which is not virtually nilpotent, $H_{b}^{2}(G)$ is infinite dimensional (List (c)).

If there are more than one piece in the torus decomposition of $M$, it suffices to discuss the following three cases.

(5) There is a piece which is a geometrically finite hyperbolic manifold.

(6) There is a piece which is a Seifert fibred space with a hyperbolic base orbifold $\Sigma$.

(7) Each piece is a Seifert fibred space with a Euclidean base orbifold.

In the case of $(5), H_{b}^{2}(G)$ is infinite dimensional. Indeed, let $H$ be one of the pieces which are hyperbolic, and $T$ one of the torus cusps of $H$. To apply List (b) to $G$, it suffices to show the following proposition (essentially shown in $[\mathrm{F} 2])$.

Proposition 3.2. $\quad$ Let $\Gamma$ be a non-elementary Kleinian group, and $P$ a parabolic subgroup. Then $|P \backslash \Gamma / P|=\infty$.

Proof. Let $p$ be the point at infinity fixed by $P$. For each element $g \in$ $\Gamma$, we have $\overline{P g P(p)}=\overline{P g(p)}$, which is a countable set since $p$ is the only accumulation point for the set $P(g(p))$. Now, if $|P \backslash \Gamma / P|$ were finite, then $\overline{\Gamma(p)}$ would be a countable set as well. It is a standard fact that $\overline{\Gamma(p)}$ coincides with 
the limit set of $\Gamma$, which is an uncountable infinite set when $\Gamma$ is non-elementary. Therefore $|P \backslash \Gamma / P|=\infty$.

In the case of $(6), H_{b}^{2}(G)$ is infinite dimensional. We can argue in the same way as (5) since the double coset space of $G$ with respect to one of the torus boundary groups $T$ is infinite. To see this, it suffices to show that the double coset space of $\pi_{1}^{\text {orb }}(O)$ with respect to the boundary subgroup $C$ (isomorphic to $\mathbb{Z}$ ) which corresponds to $T$ is infinite. Since $O$ is hyperbolic, $\pi_{1}^{\text {orb }}(O)$ is non-elementary word-hyperbolic group. We apply the following fact ([F2]). (Alternatively, one can argue using points at infinity as $(5)$ since $\pi_{1}^{\text {orb }}(O)$ is a Fuchsian group.)

Proposition 3.3. Let $\Gamma$ be a non-elementary word-hyperbolic group, and $C$ a subgroup isomorphic to $\mathbb{Z}$. Then $|C \backslash \Gamma / C|=\infty$.

In the case of (7), the 3 -manifold $M$ is obtained by pasting two twisted $S^{1}$-bundles over Möbius bands at their boundaries, and $G$ is virtually solvable. Indeed, the base orbifold of each piece has to be either an annulus, a Möbius band, or a disc with two cone points of index 2 since it is Euclidean. The latter two give manifolds which are homeomorphic to each other, but with different fibrations. Since the torus decomposition is minimal, an $S^{1}$-bundle over an annulus does not appear. Thus the only case that remains to be considered is when $M$ is a graph manifold obtained by pasting two twisted $S^{1}$-bundles over a Möbius band at their boundaries (so that the fibration cannot extend to the entire manifold). In this case, we have an exact sequence, $1 \rightarrow \mathbb{Z} \times \mathbb{Z} \rightarrow$ $\pi_{1}(M) \rightarrow \mathbb{Z}_{2} * \mathbb{Z}_{2} \rightarrow 1$. Thus $G$ contains a subgroup of index 2 which is solvable.

As a conclusion, among the cases $(1)-(7), H_{b}^{2}(G)$ is infinite dimensional in the cases of (3), (4), (5) and (6), and $G$ is virtually solvable in the cases of (1), (2) and (7), which corresponds to (i), (ii)', (iii) in Theorem 1.2. As we already discussed, the other possibilities for $G$ to be virtually solvable are when $G$ is finite or isomorphic to $\mathbb{Z}$ or $\mathbb{Z}_{2} * \mathbb{Z}_{2}$, which exactly correspond to the manifolds in (iv), (v) or (vi).

We prove Corollary 1.3.

Proof. If $M$ is reducible or boundary reducible, Theorem 1.1 applies. If $\partial M$ is not empty, then Theorem 1.2 applies. Suppose $M$ is irreducible and without boundary. Since $G$ contains a subgroup isomorphic to $\mathbb{Z} \times \mathbb{Z}, M$ either contains an embedded, incompressible torus or is homotopy equivalent to a Seifert fibred space ([Sc3], [CJu], [Ga]). In either case, Theorem 1.2 applies to $M$. 


\section{References}

[BaGh] Barge, J. and Ghys, E., Cocycles bornés et actions de groupes sur les arbres réels, Group Theory from a Geometric Viewpoint, World Sci. Pub., New Jersey, 1991, 617-622.

[Bo] Bouarich, A., Suites exactes en cohomologie bornée réelle des groupes discrets, Comptes Rendus Acad. Sci. Paris, Série I, 320 (1995), 1355-1359.

[B] Brooks, R., Some remarks on bounded cohomology, Ann. of Math. Stud., 97 (Princeton Univ Press, Princeton, 1981), 53-63.

[CJu] Casson, A. and Jungreis, D., Convergence groups and Seifert fibered 3-manifolds, Invent. Math., 118 (1994), 441-456.

[EF] Epstein, D. B. A. and Fujiwara, K., The second bounded cohomology of wordhyperbolic groups, Topology, 36 (1997), 1275-1289.

[F1] Fujiwara, K., The bounded cohomology of an amalgamated free product of groups, Trans. Amer. Math. Soc., 352 (2000), 1113-1129.

[F2] - The second bounded cohomology of a group acting on a Gromov-hyperbolic space, Proc. London Math. Soc. (3), 76 (1998), 70-94.

[Ga] Gabai, D., Convergence groups are Fuchsian groups, Ann. of Math. (2), 136 (1992), $447-510$

[G1] Gromov, M., Volume and bounded cohomology, Publ. Math. IHES, 56 (1982), 5-99.

[G2] —- Hyperbolic groups, Essays in group theory, MSRI Publ, 8, S. M. Gersten (ed)., Springer, New York, 1987, pp. 75-263.

[I] Ivanov, N. V., Foundations of the theory of bounded cohomology, Zap. Nauchn. Sem. Leningr. Otd. Mat. Inst., 143 (1985), 69-109.

[Jo] Johannson, K., Homotopy equivalences of 3-manifolds with boundaries, Springer Lecture Notes in Math., 761, Springer Verlag, Berlin-Heidelberg-New York, 1979.

[JSh] Jaco, W. and Shalen, P., Seifert fibred spaces in 3-manifolds, Mem. Amer. Math. Soc., 21 (AMS, 1979).

[MaMo] Matsumoto, S. and Morita, S., Bounded cohomology of certain groups of homeomorphisms, Proc. Amer. Math. Soc., 94 (1985), 539-544.

[Mi] Mitsumatsu, Y., Bounded cohomology and $l^{1}$-homology of surfaces, Topology, 23 (1984), 465-471.

[Sc1] Scott, P., The geometries of 3-manifolds, Bull. London Math. Soc., 15 (1983), 401487.

[Sc2] - Compact submanifolds of 3-manifolds, J. London Math. Soc. (2), 7 (1973), $246-250$.

[Sc3] - A new proof of the annulus and torus theorems, Amer. J. Math., 102 (1980), 241-277.

[T] Thurston, W. P., Three-dimensional manifolds, Kleinian groups and hyperbolic geometry, Bull. Amer. Math. Soc. (N.S.), 6 (1982), 357-381. 